\title{
OPEN Self-reported symptom study of COVID-19 chemosensory dysfunction in Malaysia
}

\begin{abstract}
Shen-Han Lee ${ }^{1 凶}$, Zhi Xiang Yeoh ${ }^{1}$, Ida Sadja'ah Sachlin ${ }^{1}$, Norzi Gazali', Shahrul Aiman Soelar ${ }^{2}$, Chee Yoong Foo ${ }^{3}$, Lee Lee Low ${ }^{4}$, Sharifah Baizura Syed Alwi', Tengku Mohamed Izam Tengku Kamalden ${ }^{5}$, Jothi Shanmuganathan ${ }^{6}$, Masliza Zaid ${ }^{7}$, Chun Yiing Wong ${ }^{8}$, Hock Hin Chua ${ }^{9}$, Suhaimi Yusuf ${ }^{10}$, Dzawani Muhamad ${ }^{11}$, Philip Rajan Devesahayam ${ }^{12}$, Hong Bee $\mathrm{Ker}^{13}$, Zulkiflee Salahuddin ${ }^{14}$, Mahiran Mustafa ${ }^{15}$, Halimuddin Sawali ${ }^{16}$, Heng Gee Lee ${ }^{17}$, Sobani $\operatorname{Din}^{18}{ }^{1}$, Nor Arisah Misnan ${ }^{19}$, Amran Mohamad ${ }^{20}$, Mohd Noor Ismail ${ }^{21}$, Chenthilnathan Periasamy ${ }^{22}$, Ting Soo Chow ${ }^{23}$, Elang Kumaran Krishnan ${ }^{24}$, Chee Loon Leong ${ }^{25}$, Linda Pei Fang Lim ${ }^{26}$, Nor Zaila Zaidan ${ }^{27}$, Mohd Zambri Ibrahim ${ }^{28}$, Suhaila Abd Wahab ${ }^{29}$, Siti Sabzah Mohd Hashim ${ }^{1}$ \& Malaysian COVID-19 Anosmia Research Group*
\end{abstract}

\begin{abstract}
Alterations in the three chemosensory modalities-smell, taste, and chemesthesis-have been implicated in Coronavirus Disease 2019 (COVID-19), yet emerging data suggest a wide geographic and ethnic variation in the prevalence of these symptoms. Studies on chemosensory disorders in COVID19 have predominantly focused on Caucasian populations whereas Asians remain understudied. We conducted a nationwide, multicentre cross-sectional study using an online questionnaire on a cohort of RT-PCR-confirmed adult COVID-19 patients in Malaysia between 6 June and 30 November 2020. The aim of our study was to investigate their presenting symptoms and assess their chemosensory function using self-ratings of perceived smell, taste, chemesthesis, and nasal blockage. In this cohort of 498 patients, $41.4 \%$ reported smell and/or taste loss when diagnosed with COVID-19, which was the commonest symptom. Blocked nose, loss of appetite, and gastrointestinal disturbances were independent predictors of smell and/or taste loss on multivariate analysis. Self-ratings of chemosensory function revealed a reduction in smell, taste, and chemesthesis across the entire cohort
\end{abstract}

\footnotetext{
${ }^{1}$ Department of Otorhinolaryngology, Hospital Sultanah Bahiyah, KM 6 Jalan Langgar, Alor Setar, 05450 Kedah, Malaysia. ${ }^{2}$ Clinical Research Centre, Hospital Sultanah Bahiyah, Kedah, Malaysia. ${ }^{3}$ Real World Insights, IOVIA Asia Pacific, Petaling Jaya, Malaysia. ${ }^{4}$ Department of Medicine, Hospital Sultanah Bahiyah, Kedah, Malaysia. ${ }^{5}$ Department of Otorhinolaryngology, Hospital Sultan Ismail, Johor, Malaysia. ${ }^{6}$ Department of Otorhinolaryngology, Hospital Sultanah Aminah, Johor, Malaysia. ${ }^{7}$ Department of Medicine, Hospital Sultanah Aminah, Johor, Malaysia. ${ }^{8}$ Department of Otorhinolaryngology, Hospital Umum Sarawak, Sarawak, Malaysia. ${ }^{9}$ Department of Medicine, Hospital Umum Sarawak, Sarawak, Malaysia. ${ }^{10}$ Department of Otorhinolaryngology, Hospital Tengku Ampuan Afzan, Pahang, Malaysia. ${ }^{11}$ Department of Medicine, Hospital Tengku Ampuan Afzan, Pahang, Malaysia. ${ }^{12}$ Department of Otorhinolaryngology, Hospital Raja Permaisuri Bainun, Perak, Malaysia. ${ }^{13}$ Department of Medicine, Hospital Raja Permaisuri Bainun, Perak, Malaysia. ${ }^{14}$ Department of Otorhinolaryngology, Hospital Raja Perempuan Zainab II, Kelantan, Malaysia. ${ }^{15}$ Department of Medicine, Hospital Raja Perempuan Zainab II, Kelantan, Malaysia. ${ }^{16}$ Department of Otorhinolaryngology, Hospital Queen Elizabeth, Sabah, Malaysia. ${ }^{17}$ Department of Medicine, Hospital Queen Elizabeth, Sabah, Malaysia. ${ }^{18}$ Department of Otorhinolaryngology, Hospital Sungai Buloh, Selangor, Malaysia. ${ }^{19}$ Department of Medicine, Hospital Sungai Buloh, Selangor, Malaysia. ${ }^{20}$ Department of Otorhinolaryngology, Hospital Sultanah Nur Zahirah, Terengganu, Malaysia. ${ }^{21}$ Department of Medicine, Hospital Sultanah Nur Zahirah, Terengganu, Malaysia. ${ }^{22}$ Department of Otorhinolaryngology, Hospital Pulau Pinang, Penang, Malaysia. ${ }^{23}$ Department of Medicine, Hospital Pulau Pinang, Penang, Malaysia. ${ }^{24}$ Department of Otorhinolaryngology, Hospital Kuala Lumpur, Kuala Lumpur, Malaysia. ${ }^{25}$ Department of Medicine, Hospital Kuala Lumpur Hospital, Kuala Lumpur, Malaysia. ${ }^{26}$ Department of Otorhinolaryngology, Hospital Melaka, Melaka, Malaysia. ${ }^{27}$ Department of Medicine, Hospital Melaka, Melaka, Malaysia. ${ }^{28}$ Department of Otorhinolaryngology, Hospital Tuanku Fauziah, Perlis, Malaysia. ${ }^{29}$ Department of Medicine, Hospital Tuanku Fauziah, Perlis, Malaysia. *A list of authors and their affiliations appears at the end of the paper. ${ }^{\square}$ email: shen-han.lee@cantab.net
} 
of patients that was more profound among those reporting smell and/or taste loss as their presenting symptom. Perceived nasal obstruction accounted for only a small proportion of changes in smell and taste, but not for chemesthesis, supporting viral disruption of sensorineural mechanisms as the dominant aetiology of chemosensory dysfunction. Our study suggests that chemosensory dysfunction in COVID-19 is more widespread than previously reported among Asians and may be related to the infectivity of viral strains.

Study Registration: NMRR-20-934-54803 and NCT04390165.

Chemosensory disorders-encompassing smell, taste, and chemesthesis-are increasingly recognised as important symptoms of the Coronavirus Disease 2019 (COVID-19) infection caused by the Severe Acute Respiratory Syndrome Coronavirus 2 (SARS-CoV-2). While early cohort studies reported the most prevalent symptoms to be fever, dry cough, dyspnoea, myalgia, diarrhoea, and sore throat ${ }^{1,2}$, several self-reported symptom studies mainly from the United States, United Kingdom, and Europe have reported smell and taste loss with a prevalence as high as $60-80 \%^{3-7}$. The link between COVID-19 and altered chemesthesis-the ability to detect chemically triggered sensations such as spiciness, burning, cooling, or tingling sensation via the trigeminal nerve-was described in a large-scale online survey study by the Parma et al. from the Global Consortium for Chemosensory Research $(\mathrm{GCCR})^{8}$.

The extent to which findings from these studies, conducted predominantly in Caucasian populations, can be applied to other populations with differences in genetics, lifestyle, environmental, and cultural factors-as well as differences in infectivity of SARS-CoV-2 genetic variants-remain largely understudied. Understanding the extent of chemosensory disorders within other populations may offer insights into the infectivity of viral strains as well as aid the diagnosis and management of the COVID-19 pandemic within a particular region. A limited number of studies from Asia-derived mainly from health records rather than self-reporting of symptoms-have reported a much lower prevalence of smell and taste loss in COVID-19 (as low as 5\%) ${ }^{9-11}$ while no study has reported chemesthesis loss in Asians. A meta-analysis by Von Bartheld et al. found a three-fold higher prevalence of smell and taste loss in Caucasians (54.8\%) as compared to Asians (17.7\%). The difference in prevalence was postulated to be due to geographical differences in the distribution of viral strains harbouring the more infectious D614G spike protein mutation and ethnic-specific differences in genetic variants of viral binding proteins, angiotensin-converting enzymes 2 (ACE-2) and transmembrane protease serine 2 (TMPRSS2) ${ }^{12,13}$.

Here, we report findings from a cross-sectional study that assessed smell, taste, and chemesthesis disturbances in a cohort of COVID-19 patients in Malaysia. Our primary aim was to investigate the timing, severity, qualitative, and quantitative changes of chemosensory function before and during COVID-19. Our secondary aims were to uncover independent predictors of loss of smell and/or taste in COVID-19 and to assess the relationship between changes in smell, taste, chemesthesis, and self-perceived nasal blockage.

\section{Methods}

Study design. This is a cross-sectional study involving 14 COVID-19 treating public hospitals across all states of Malaysia. A self-administered questionnaire was used to survey patients diagnosed with COVID-19 infection in Malaysia between 6 June and 30 November 2020.

Patient eligibility. A convenience sample of patients aged $\geq 18$ years with COVID-19 infection confirmed with reverse transcription polymerase chain reaction were invited to participate in the survey. Participants were either inpatient or patients who were discharged back to the community at the time of survey administration. Inpatient participants were evaluated to be clinically stable prior to initiating the survey. Those who were in the intensive care unit at the time of study were excluded. An internet link to the questionnaire online was sent to an invited patient after they have given verbal and written informed consent to participate. Phone interviews were conducted to those who did not have internet access or who were illiterate.

Ethics approval. This study received ethics approval from the Medical Research and Ethics Committee, Ministry of Health, Malaysia (KKM/NIHSEC/P20-1112). All methods in this study were performed in accordance with the relevant guidelines and regulations.

Questionnaire design. The survey questionnaire was adapted from a pre-existing, validated online questionnaires developed by the $\mathrm{GCCR}^{8}$ and the American Academy of Otolaryngology-Head \& Neck Surgery (AAO-HNS $)^{7}$. After multiple iterations, a consensus on the final version of the questionnaire was reached among the investigators and satisfied adequate content and face validity per our local setting. Our questionnaire was available in Malay and English, two of the widest spoken languages in Malaysia (Supplementary Materials S1). Participants were asked to report demographic information, symptoms of their COVID-19 diagnosis, time of onset of smell and/or taste loss, severity of symptoms, and whether they had recovered from them at the time of filling the questionnaire. They were given the option to describe any specific changes in smell and taste qualities as a check-all-that applies (CATA) question. They were also asked to quantify their ability to smell, taste, and perceive cooling, tingling and burning sensations (chemesthesis), and perceived nasal obstruction before, during COVID-19, and at the time of filling the questionnaire on a 6-point visual analogue scale (VAS). 
Sample size calculation. Sample size estimation was calculated using the population proportion formula ${ }^{14}$. Prior data indicate that the prevalence of COVID-19-associated chemosensory dysfunction was $47.4 \%^{12}$. With a Type I error probability of 5\%, precision of 5\%, and an estimated prevalence of $50 \%$, we will need to study 384 samples. With an additional $20 \%$ dropout rate, the sample size needed was 480 samples.

Statistical analyses. Associations between categorical variables were tested using Chi-Square test, while differences in mean age were tested using an independent sample $t$-test. Variables associated with smell and taste disturbances were first tested using univariate analysis, and statistically significant variables were then tested in a multivariate logistic regression analysis. The relationship between the categories of symptom severity and the presence of hyposmia or anosmia was tested using Chi-squared test with post hoc Bonferroni correction. The relationship between the categories of symptom severity and self-ratings during COVID-19 diagnosis was tested using Spearman's rank correlation analysis. Self-ratings of smell, taste, chemesthesis, and nasal congestion before and during COVID-19 diagnosis were tested with Wilcoxon matched pairs signed-rank test. A level of $\mathrm{p}<0.05$ was considered statistically significant. All statistical analyses were performed using either GraphPad PRISM 9.0 (GraphPad Software Inc., San Diego, CA, USA) or IBM SPSS Statistics (SPSS) version 27.0 (IBM Corporation, Armonk, NY, USA). Principal component analysis (PCA) of the changes in self-ratings of smell, taste, chemesthesis, and nasal blockage [(rating during COVID-19 diagnosis) minus (rating before COVID-19 diagnosis)] was performed in the same manner as previously reported by Parma et al. ${ }^{8}$ using the prcomp function from the $\mathrm{R}$ default statistics package. PCA is an algorithm that reduces the dimensionality of a dataset while retaining most of the variation in the dataset by identifying directions-termed principal components-along which variation of the data is maximal. Results of the PCA were plotted using functions from the FactoMineR package ${ }^{15}$.

\section{Results}

Patient recruitment and characteristics. A sample of 827 eligible patients were invited to complete the questionnaire, 743 agreed to participate, and 532 responses were received (response rate 64.3\%). 34 responses were excluded due to either not meeting the inclusion criteria, duplicate responses, or inconsistent responses, while the remaining 498 responses were analysed.

Overall, the age of the patients ranged from 18 to 87 (median \pm interquartile range [IQR]: $36 \pm 24.25$ years old). There were 279 males (56\%) and 219 (44\%) females. The largest ethnic group in our cohort were Malays (76.7\%), followed by Chinese (10.0\%) and Indians (1.8\%), while the remaining (11.5\%) included several ethnic groups native to East Malaysia (e.g., Kadazan, Dusun, and Murut) and foreign nationals (3\%). 54.4\% of patients had at least one comorbidity, the most common being hypertension (17.3\%) and diabetes mellitus (13.9\%) (Table 1).

Prevalence, timing, and severity of smell and taste disorders. At time of COVID-19 testing, 206 patients (41.4\%) reported either one of loss of smell and/or taste. Among them, 29.3\% reported loss of both smell and taste, $7.4 \%$ reported loss of smell but not taste, and 5.0\% reported loss of taste but not smell. Loss of smell and/or taste was the most common symptom besides fever (40.2\%), ahead of cough (30.5\%) and sore throat (25.5\%) (Table 1). $34.6 \%$ of patients with smell loss reported experiencing this before other symptoms whereas $30.7 \%$ of patients with taste loss experienced this before other symptoms (Table 2). $7.7 \%$ and $3.0 \%$ of patients with smell and taste loss respectively experienced this as their only symptom.

In terms of symptom severity, $12.1 \%$ and $10.0 \%$ of patients with smell and taste loss respectively described their symptoms as "severe" to "as bad as it can be" in the preceding two weeks prior to diagnosis (Table 2). In the CATA question on the type of smell disorders, 73 patients (40.1\%) reported complete loss of smell (anosmia) whereas 100 patients (55.5\%) reported partial loss of smell (hyposmia). Of note, 17 patients (9.3\%) reported fluctuating sense of smell (Table 2). There was no significant correlation between the six categories of symptom severity and the presence of anosmia or hyposmia, as determined by Chi-square test with post hoc Bonferroni correction $[Z$ critical value $=2.86$, adjusted alpha level $=0.004(0.05 / 12)]$. In addition, there was no correlation between these categories of symptom severity and changes in rating scores of the patients when diagnosed with COVID-19 [Spearman's rank correlation coefficient, $\mathrm{r}=0.05, \mathrm{p}=0.5$ ].

Factors predictive of smell and taste disorders. Loss of smell and/or taste were significantly associated with younger age group ( $<50$ years), female sex, and the presence of several other symptoms listed in Table 3 on univariate analyses. A multivariate logistic regression analysis was performed using these variables and found that the presence of blocked nose ( $\mathrm{p}<0.0001$, OR 4.95, CI 2.41-10.15), loss of appetite $(\mathrm{p}<0.0001$, OR 4.16, CI 2.35-7.38), and gastrointestinal disturbances ( $\mathrm{p}=0.038$, OR 2.17, CI 1.04-4.53) were independent predictors of loss of smell and/or taste (Table 3).

Quantitative changes of smell, taste, chemesthesis, and nasal obstruction during COVID-19. The distribution of patients' self-ratings of smell, taste, and chemesthesis, and nasal obstruction before and during COVID-19 diagnosis are depicted in Fig. 1. There were statistically significant changes in selfratings of smell, taste, chemesthesis, and nasal blockage in the total patient cohort and the subgroups before and during COVID-19 diagnosis as measured by Wilcoxon matched pairs signed-rank test (Table 4).

We observed an increase in the percentage of patients with smell, taste, and chemesthesis ratings lower than a cut-off point of 4 compared to their baseline ratings prior to COVID-19 diagnosis (smell: $35.6 \%$ from 10.4\%; taste: $34.2 \%$ from $10.0 \%$; chemesthesis: $38.7 \%$ from $22.8 \%$ ) (Fig. 1). Subgroup analysis of only those who reported smell loss as their presenting complaint $(n=182)$ revealed a higher increase in the proportion of smell ratings lower than 4 from $19.6 \%$ to $86.7 \%$. Similarly, those who reported taste loss as their presenting complaint 


\begin{tabular}{|c|c|c|}
\hline Characteristics & Total $(\mathrm{N}=498)$ & Proportion (\%) \\
\hline \multicolumn{3}{|l|}{ Age, years } \\
\hline Median (IQR) & $36(28-52)$ & \\
\hline \multicolumn{3}{|l|}{ Sex } \\
\hline Female & 219 & 43.9 \\
\hline Male & 279 & 56.0 \\
\hline \multicolumn{3}{|l|}{ Ethnicity } \\
\hline Malay & 382 & 76.7 \\
\hline Chinese & 50 & 9.8 \\
\hline Indian & 9 & 1.8 \\
\hline Other Malaysian ethnics & 47 & 9.4 \\
\hline Other nationalities & 10 & 2.0 \\
\hline \multicolumn{3}{|l|}{ Pre-existing comorbidities } \\
\hline Hypertension & 86 & 17.3 \\
\hline Diabetes & 69 & 13.9 \\
\hline Smoker & 56 & 11.2 \\
\hline Allergies/allergic rhinitis & 35 & 7.0 \\
\hline Obstructive sleep apnoea & 28 & 5.6 \\
\hline Rhinosinusitis & 27 & 5.4 \\
\hline Chronic lung disease/asthma & 24 & 4.8 \\
\hline Obesity & 23 & 4.6 \\
\hline Cardiac disease & 13 & 2.6 \\
\hline Psychiatric disorders & 7 & 1.4 \\
\hline Dyslipidaemia & 5 & 1.0 \\
\hline Previous sinonasal surgery & 5 & 1.0 \\
\hline History of head trauma & 4 & 0.8 \\
\hline Previous head/brain surgery & 4 & 0.8 \\
\hline None & 227 & 45.6 \\
\hline \multicolumn{3}{|l|}{ Presenting symptoms } \\
\hline Loss of smell \&/or taste & 206 & 41.4 \\
\hline Loss of smell & 182 & 36.6 \\
\hline Loss of taste & 169 & 33.9 \\
\hline Fever & 200 & 40.2 \\
\hline Cough & 152 & 30.5 \\
\hline Sore throat & 127 & 25.5 \\
\hline Malaise & 119 & 23.9 \\
\hline Loss of appetite & 108 & 21.7 \\
\hline Muscle ache & 75 & 15.1 \\
\hline Headache & 66 & 13.3 \\
\hline Nasal congestion & 61 & 12.2 \\
\hline Shortness of breath & 57 & 11.5 \\
\hline Rhinorrhoea & 34 & 6.8 \\
\hline None & 138 & 27.7 \\
\hline
\end{tabular}

Table 1. Demographic and clinical characteristics of COVID-19 patients assessed for olfactory \& taste disturbances.

$(\mathrm{n}=169)$ had a greater increase in the proportion of taste and chemesthesis ratings below 4 (taste, $82.9 \%$ from 16.1\%; chemesthesis: $71.1 \%$ from $26 \%$ ).

In parallel, we observed a slight increase in perceived nasal obstruction related to COVID-19. At baseline, $21 \%$ of patients reported a nasal blockage rating of greater than 1 , which increased to $27.7 \%$ when diagnosed with COVID-19. Subgroup analysis of only patients who reported smell loss as their presenting symptom $(n=182)$ found $47.8 \%$ reporting a nasal blockage rating of greater than 1 , from $27.4 \%$ at baseline. This observation is concordant with findings of significant association of smell loss with nasal congestion on multivariate analysis.

Relationship between self-ratings of smell, taste, chemesthesis, and nasal obstruction. To further characterise the relationship between changes in perceived nasal obstruction and changes in the three chemosensory modalities, we performed a principal component analysis of the changes in self-ratings of smell, taste, chemesthesis, and perceived nasal blockage (during minus before diagnosis of COVID-19) (Fig. 2). This 


\begin{tabular}{|c|c|c|}
\hline Characteristics & No & Proportion (\%) \\
\hline Loss of smell ${ }^{\mathrm{A}}$ & 182 & 36.5 \\
\hline Loss of taste & 169 & 33.9 \\
\hline Loss of smell and taste & 145 & 29.1 \\
\hline Loss of smell and/or taste & 206 & 41.4 \\
\hline Loss of smell without loss of taste & 37 & 7.4 \\
\hline Loss of taste without loss of smell & 24 & 4.8 \\
\hline \multicolumn{3}{|l|}{ Timing of loss of smell ${ }^{B}$} \\
\hline First symptom & 63 & 34.6 \\
\hline Same time as other symptoms & 49 & 26.9 \\
\hline After other symptoms & 56 & 30.7 \\
\hline Only symptom & 14 & 7.7 \\
\hline \multicolumn{3}{|l|}{ Timing of loss of taste ${ }^{\mathrm{C}}$} \\
\hline First symptom & 52 & 30.7 \\
\hline Same time as other symptoms & 47 & 27.8 \\
\hline After other symptoms & 65 & 38.4 \\
\hline Only symptom & 5 & 3 \\
\hline \multicolumn{2}{|c|}{ Decreased sense of smell 2 weeks before diagnosis } & B \\
\hline No problem & 83 & 45.6 \\
\hline Very mild problem & 20 & 11 \\
\hline Mild or slight problem & 38 & 20.9 \\
\hline Moderate problem & 19 & 10.4 \\
\hline Severe problem & 10 & 5.5 \\
\hline Problem is as bad as it can be & 12 & 6.6 \\
\hline \multicolumn{2}{|c|}{ Decreased sense of taste 2 weeks before diagnosis } & C \\
\hline No problem & 78 & 46.1 \\
\hline Very mild problem & 21 & 12.4 \\
\hline Mild or slight problem & 35 & 20.7 \\
\hline Moderate problem & 18 & 10.7 \\
\hline Severe problem & 9 & 5.3 \\
\hline Problem is as bad as it can be & 8 & 4.7 \\
\hline \multicolumn{3}{|l|}{ Type of smell disturbance ${ }^{B}$} \\
\hline Anosmia & 73 & 40.1 \\
\hline Hyposmia & 100 & 55.5 \\
\hline Parosmia & 28 & 15.3 \\
\hline Cacosmia & 13 & 7.1 \\
\hline Phantosmia & 19 & 10.4 \\
\hline Fluctuating sense of smell & 17 & 9.3 \\
\hline \multicolumn{3}{|l|}{ Type of taste disturbance ${ }^{\mathrm{C}}$} \\
\hline Sweet & 83 & 49.1 \\
\hline Salty & 90 & 53.2 \\
\hline Sour & 75 & 44.4 \\
\hline Bitter & 72 & 42.6 \\
\hline Umami & 48 & 28.4 \\
\hline
\end{tabular}

Table 2. Characteristics of olfactory and taste disturbances in COVID-19 patients. ${ }^{\mathrm{A}}$ Calculated as a proportion of total number of patients $(\mathrm{N}=498) .{ }^{\mathrm{B}}$ Calculated as a proportion of total number of patients with loss of smell $(\mathrm{n}=182) .{ }^{\mathrm{C}}$ Calculated as a proportion of total number of patients with loss of taste $(\mathrm{n}=169)$.

analytic approach was previously employed by Parma et al. ${ }^{8}$ to determine whether changes in chemosensory function can be attributed to nasal obstruction. It leverages the orthogonal features of these principal components to evaluate the degree of statistical dependence between changes in chemosensory ability and perceived nasal obstruction. In our analysis, the two orthogonal principal components, Components 1 and 2, accounted for $59 \%$ and $22 \%$ of the total multidimensional variances respectively. Changes in self-ratings of smell, taste, and chemesthesis clustered together and correlated strongly with Component 1 (smell: $r=0.837$, taste: $r=0.871$, chemesthesis: $r=0.815$ ), while showing negligible to weak positive correlation with the Component 2 (smell: $r=0.066$, taste: 0.097 , and chemesthesis: 0.333 ). In contrast, changes in self-ratings of nasal obstruction demonstrated only a moderate negative correlation with Component $1(\mathrm{r}=-0.474)$ but strong positive correlation $(r=0.873)$ with Component 2. The PCA loading vectors for changes in chemesthesis and nasal obstruction 


\begin{tabular}{|c|c|c|c|c|c|c|}
\hline \multirow[b]{2}{*}{ Variables } & \multicolumn{2}{|c|}{ Smell \&/or taste disturbance } & \multicolumn{2}{|l|}{ Univariate analysis } & \multicolumn{2}{|l|}{ Multivariate analysis } \\
\hline & Present, $\mathbf{n}(\%)$ & Absent, $\mathbf{n}(\%)$ & Odds ratio $(95 \% \mathrm{CI})$ & $p$-value ${ }^{A}$ & Odds ratio $(95 \% \mathrm{CI})$ & $p$-value ${ }^{\mathrm{B}}$ \\
\hline \multicolumn{7}{|l|}{ Age group (years) } \\
\hline$\leq 50$ & $163(45.0)$ & $199(55.0)$ & $1.77(1.17-2.72)$ & 0.007 & $1.30(0.82-2.08)$ & 0.27 \\
\hline$>50$ & $43(31.6)$ & $93(68.4)$ & 1.00 (reference) & & 1.00 (reference) & \\
\hline \multicolumn{7}{|l|}{ Sex } \\
\hline Male & $103(36.9)$ & $176(63.1)$ & $0.6591(0.46-0.94)$ & 0.02 & $0.71(0.47-1.07)$ & 0.10 \\
\hline Female & $103(47.0)$ & $116(53.0)$ & 1.00 (reference) & & 1.00 (reference) & \\
\hline \multicolumn{7}{|l|}{\begin{tabular}{|l|} 
Ethnicity \\
\end{tabular}} \\
\hline Malay & $166(43.5)$ & $216(56.5)$ & $1.46(0.95-2.26)$ & 0.09 & - & - \\
\hline Chinese & $20(40.0)$ & $30(60.0)$ & $0.94(0.52-1.69)$ & 0.84 & - & - \\
\hline Indian & $5(55.6)$ & $4(44.4)$ & $1.94(0.58-6.37)$ & 0.32 & - & - \\
\hline Other Malaysian ethnics & $15(31.9)$ & $32(68.1)$ & $0.59(0.31-1.13)$ & 0.10 & - & - \\
\hline \multicolumn{7}{|l|}{ Comorbidities } \\
\hline Hypertension & $32(37.2)$ & $54(62.8)$ & $0.81(0.51-1.29)$ & 0.40 & - & - \\
\hline Diabetes & $25(36.2)$ & $44(63.8)$ & $0.78(0.46-1.31)$ & 0.43 & - & - \\
\hline Smoking & $21(37.5)$ & $35(62.5)$ & $0.83(0.48-1.48)$ & 0.57 & - & - \\
\hline Allergies/allergic rhinitis & $16(45.7)$ & $19(54.3)$ & $1.21(0.63-2.41)$ & 0.60 & - & - \\
\hline Obstructive sleep apnoea & $14(50.0)$ & $14(50.0)$ & $1.45(0.68-3.05)$ & 0.43 & - & - \\
\hline \begin{tabular}{|l|} 
Rhinosinusitis \\
\end{tabular} & $12(44.4)$ & $15(55.6)$ & $1.16(0.53-2.59)$ & 0.84 & - & - \\
\hline Chronic lung disease/asthma & $14(53.9)$ & $12(46.2)$ & $1.70(0.80-3.78)$ & 0.22 & - & - \\
\hline Obesity & $12(52.2)$ & $11(47.8)$ & $1.41(0.59-3.10)$ & 0.52 & - & - \\
\hline Cardiac disease & $6(46.2)$ & $7(53.9)$ & $1.22(0.40-3.34)$ & 0.78 & - & - \\
\hline Psychiatric disorders & $3(42.8)$ & $4(57.1)$ & $1.06(0.27-4.00)$ & $>0.99$ & - & - \\
\hline Previous sinonasal surgery & $3(42.8)$ & $4(57.1)$ & $1.06(0.27-4.00)$ & $>0.99$ & - & - \\
\hline History of head trauma & $1(25.0)$ & $3(75.0)$ & $1.66(0.59-9.12)$ & 0.65 & - & - \\
\hline Previous head/brain surgery & $1(20.0)$ & $4(80.0)$ & $0.35(0.03-2.14)$ & 0.65 & - & - \\
\hline \multicolumn{7}{|l|}{ Associated symptoms } \\
\hline Fever & $110(55)$ & $90(45)$ & $2.53(1.78-3.69)$ & $<0.001$ & $1.47(0.92-2.348)$ & 0.11 \\
\hline Cough & $74(48.7)$ & $78(51.3)$ & $1.54(1.03-1.57)$ & 0.03 & $0.64(0.39-1.05)$ & 0.08 \\
\hline Sore throat & $74(58.3)$ & $53(41.7)$ & $2.53(1.67-3.80)$ & $<0.001$ & $1.53(0.93-2.54)$ & 0.10 \\
\hline Malaise & $72(60.5)$ & 47 (39.5) & $2.80(1.81-4.24)$ & $<0.001$ & $0.93(0.52-1.68)$ & 0.81 \\
\hline Loss of appetite & $79(73.8)$ & $28(26.2)$ & $5.87(3.64-9.59)$ & $<0.001$ & $4.17(2.35-7.38)$ & $<0.001$ \\
\hline Muscle ache & $43(57.3)$ & $32(42.7)$ & $2.14(1.29-3.55)$ & 0.003 & $0.71(0.36-1.39)$ & 0.32 \\
\hline Headache & $46(69.7)$ & $20(30.3)$ & $3.91(2.21-6.71)$ & $<0.001$ & $1.40(0.68-2.89)$ & 0.36 \\
\hline Nasal congestion & $46(76.7)$ & $14(23.3)$ & $5.71(3.02-10.37)$ & $<0.001$ & $4.95(2.41-10.15)$ & $<0.001$ \\
\hline Rhinorrhoea & $22(64.7)$ & $12(35.3)$ & $2.79(1.36-5.64)$ & 0.004 & $1.24(0.52-2.97)$ & 0.63 \\
\hline Chills & $20(64.5)$ & $11(35.5)$ & $2.75(1.26-6.04)$ & 0.007 & $1.48(0.60-3.69)$ & 0.40 \\
\hline Gastrointestinal disturbances & $44(73.3)$ & $16(26.7)$ & $4.69(2.56-8.52)$ & $<0.001$ & $2.17(1.04-4.53)$ & 0.04 \\
\hline
\end{tabular}

Table 3. Factors associated with smell and/or taste disturbances among COVID-19 patients in Malaysia. Bold values indicate statistical significance $(\mathrm{p}<0.05)$. $(-)$ not included in multivariate model. ${ }^{\mathrm{A}} \mathrm{p}$-values were calculated using Chi square test (or Fisher's exact test when $\mathrm{n}<5$ in any cell). ${ }^{\mathrm{B}} \mathrm{p}$-values for multivariate analysis were calculated using multiple logistic regression (Forward method).

formed a right angle indicating that they were not correlated and statistically independent of each other, whereas vectors for smell and taste changes formed a small obtuse angle with the vector for nasal obstruction, indicating a weak negative correlation. These PCA findings suggest that nasal obstruction could only account for a small proportion of smell and taste changes, but not for chemesthesis.

Qualitative changes of smell and taste during COVID-19. Qualitative changes in smell were uncommon among those who experienced smell disturbances-only 28 patients $(15.3 \%)$ experienced parosmia, 19 patients (10.4\%) experienced phantosmia, and 13 patients (7.1\%) experienced cacosmia. The distinction between parosmia and cacosmia were made on the basis of patients' selected response to Question 27 of the Questionnaire (Supplementary Material S1). Patients who selected "Smells smell different than they did before (the quality of smell has changed)" were considered to have parosmia, whereas those who selected "Smells smell unpleasant" were considered to have cacosmia.

Among participants who reported gustatory changes, 33 patients (19.5\%) reported impairment of a single taste quality and 106 patients $(62.7 \%)$ reported impairment of 2 or more taste qualities in the CATA question. 30 
A

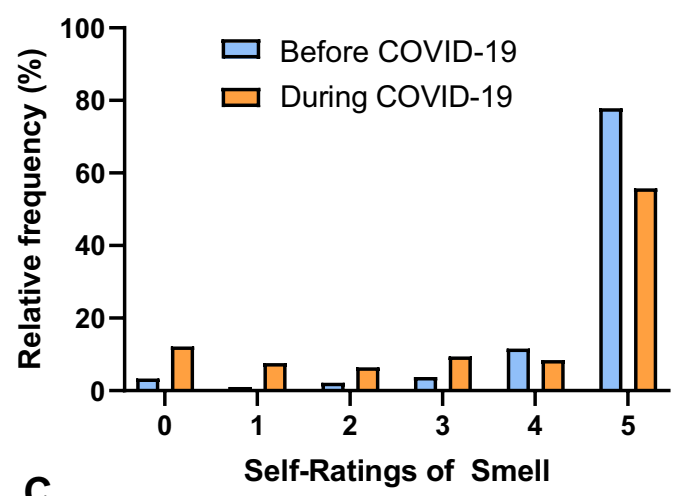

C

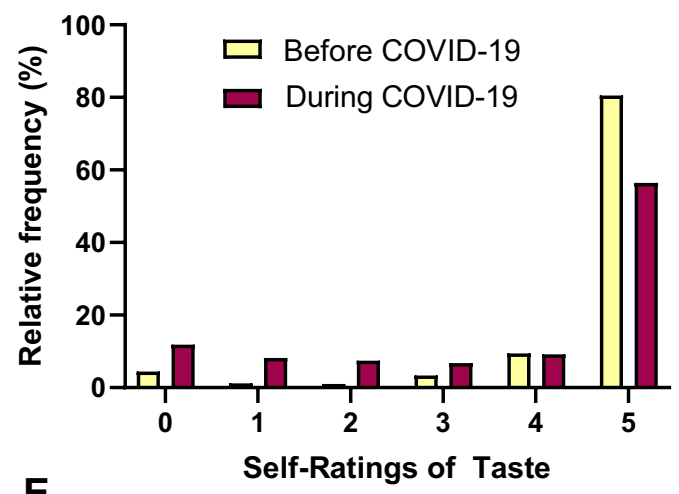

E

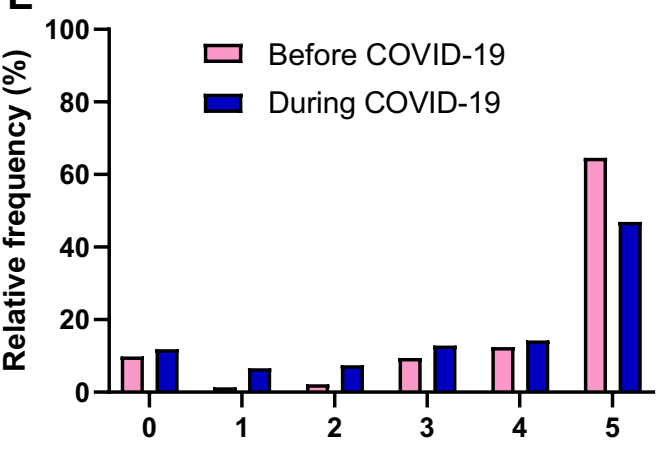

G

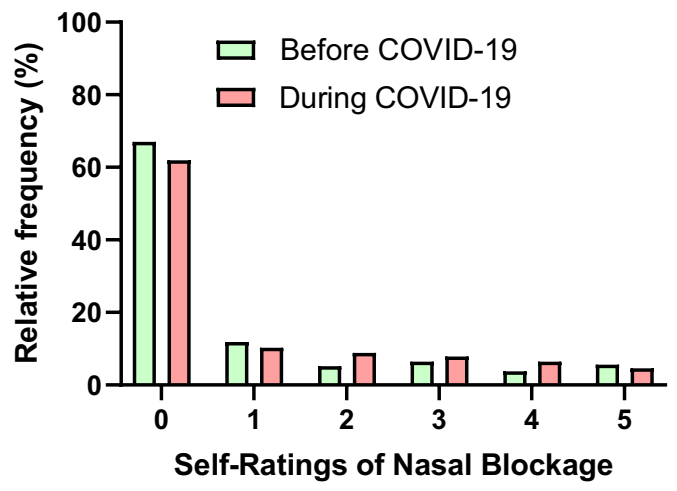

B
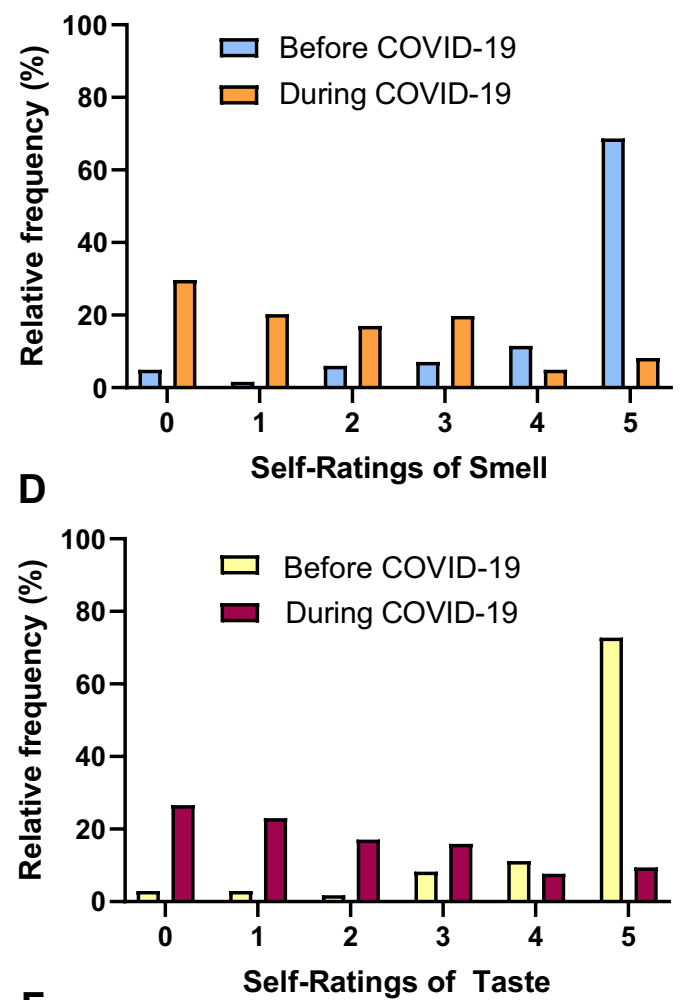

$\mathbf{F}$

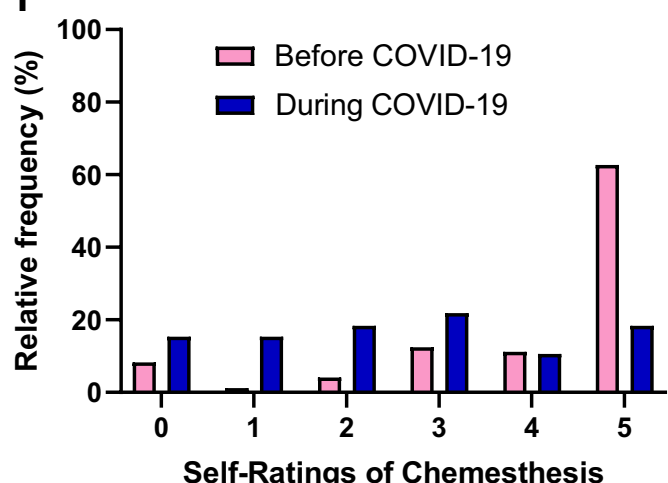

H

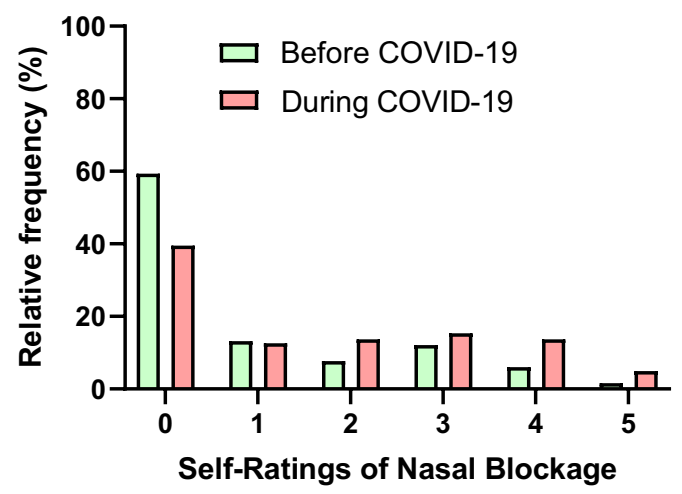

Figure 1. Self-ratings of smell, taste, chemesthesis, and nasal congestion before and during COVID-19. Interleaved histograms represent self-ratings for smell, taste, chemesthesis, and nasal congestion on a 6-point visual analogue scale before and during COVID-19 in all patients (A,C,E,G), and the subset of patients who report changes in smell $(\mathbf{B}, \mathbf{H})$ and taste $(\mathbf{D}, \mathbf{F})$. 


\begin{tabular}{|c|c|c|c|c|c|c|c|}
\hline \multirow[b]{2}{*}{ Variable } & \multicolumn{2}{|c|}{\begin{tabular}{|l|} 
Before \\
COVID-19
\end{tabular}} & \multicolumn{2}{|c|}{$\begin{array}{l}\text { During } \\
\text { COVID-19 }\end{array}$} & \multicolumn{2}{|c|}{ Ratings below/above cut-off score (\%) ${ }^{A}$} & \multirow[b]{2}{*}{ p-value ${ }^{\mathrm{I}}$} \\
\hline & Mean & SE & Mean & SE & Before COVID-19 & During COVID-19 & \\
\hline \multicolumn{8}{|l|}{ Smell ratings } \\
\hline All patients & 4.53 & 0.05 & 3.62 & 0.08 & 10.4 & 35.6 & $<0.001$ \\
\hline \begin{tabular}{|l|} 
Smell disturbance only \\
\end{tabular} & 4.25 & 0.10 & 1.74 & 0.12 & 19.6 & 86.7 & $<0.001$ \\
\hline \multicolumn{8}{|l|}{ Taste ratings } \\
\hline All patients & 4.54 & 0.05 & 3.63 & 0.08 & 10.0 & 34.2 & $<0.001$ \\
\hline Taste disturbance only & 4.40 & 0.09 & 1.83 & 0.12 & 16.1 & 82.9 & $<0.001$ \\
\hline \multicolumn{8}{|l|}{\begin{tabular}{|l|} 
Chemesthesis ratings \\
\end{tabular}} \\
\hline All patients & 4.07 & 0.07 & 3.52 & 0.08 & 22.8 & 38.7 & $<0.001$ \\
\hline Taste disturbance only & 4.05 & 0.12 & 2.52 & 0.13 & 26.0 & 71.1 & $<0.001$ \\
\hline \multicolumn{8}{|l|}{ Nasal blockage ratings } \\
\hline All patients & 0.85 & 0.07 & 1.00 & 0.07 & 21.0 & 27.7 & $<0.01$ \\
\hline Smell disturbance only & 0.97 & 0.10 & 1.66 & 0.12 & 27.4 & 47.8 & $<0.001$ \\
\hline
\end{tabular}

Table 4. Self-ratings of smell, taste, chemesthesis, and nasal obstruction before and during COVID-19. ${ }^{\mathrm{A}}$ For smell, taste and chemesthesis, ratings are below the cut-off score of 4 . For nasal blockage, ratings are above the cut-off score of $1 .{ }^{\mathrm{B}}$ Wilcoxon matched pairs signed-rank test.

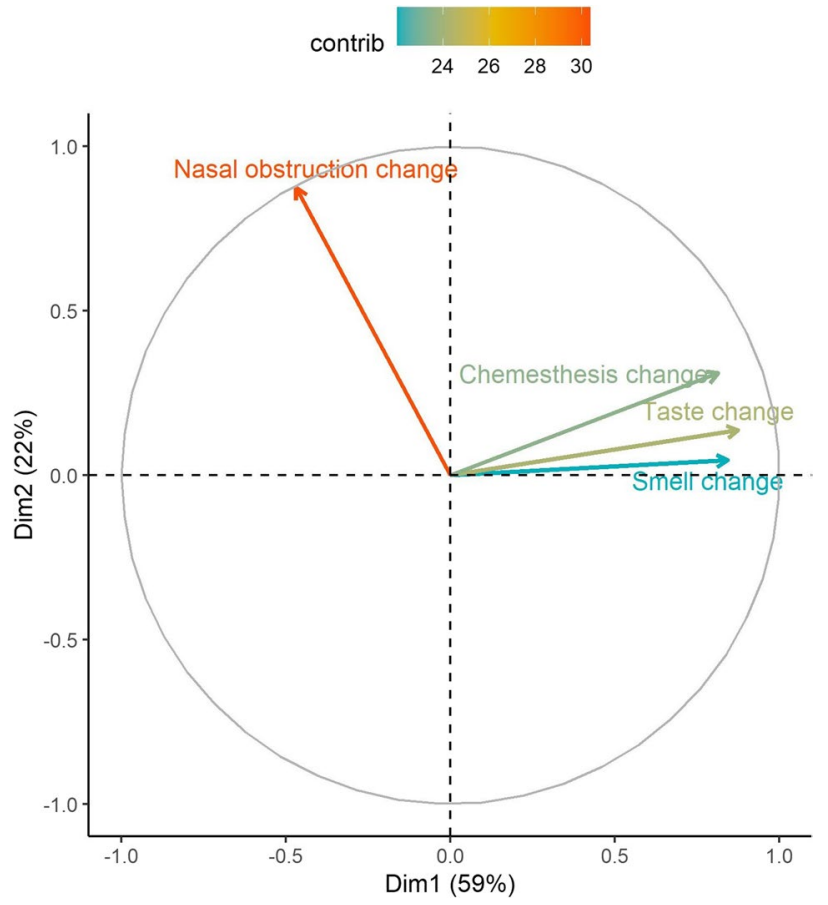

Figure 2. Principal component analysis (PCA) of difference scores of ratings of smell, taste, and chemesthesis [(rating during COVID-19 diagnosis) minus (rating before COVID-19 diagnosis)]. The findings of the PCA are depicted by a correlation circle of vectors representing changes in perceived smell, taste, chemesthesis and nasal blockage with the first (Dimension 1, abscissa) and second (Dimension 2, ordinate) principal components.

patients $(17.8 \%)$ did not respond to this question (Table 2 ). Salty taste was the most frequently reported change (53.2\%) followed by sweet $(49.1 \%)$, sour (44.4\%), bitter (42.6\%), and umami/savoury (28.4\%) taste.

Recovery of smell and taste disorders. Among the patients with smell and/or taste disorders, $90.2 \%$ $(n=186)$ of them reported recovery of their symptoms at the time of answering the questionnaire. This was on average $113 \pm 31$ days (mean \pm SD) from the date of their COVID-19 diagnosis. Self-ratings of sense of smell, taste, chemesthesis, and nasal congestion at this time-point returned to pre-COVID-19 baseline levels in all patients and in the subset of patients who reported the chemosensory disorders [Mean smell rating: all patients $4.66 \pm 0.86$; smell disturbance only $4.49 \pm 0.93$; mean taste rating: all patients $4.74 \pm 0.78$; taste disturbance only 
$4.61 \pm 0.82$; mean chemesthesis rating: all patients $4.16 \pm 1.51$; taste disturbance only $4.20 \pm 1.36$; mean nasal congestion rating: all patients $0.54 \pm 1.22$; smell disturbance only $0.60 \pm 1.23$; mean $\pm \mathrm{SD}$ ].

\section{Discussion}

Our survey which involved close to 500 patients treated in 14 COVID-19-treating public hospitals in Malaysia revealed that loss of smell and/or taste was not only an early symptom of COVID-19 infection, but also the commonest symptom in this cohort, involving $40.2 \%$ of our patients. In comparison, a previous multicentre nationwide study from Malaysia based on the health records of 5889 hospitalised patients found the most common clinical manifestation of COVID-19 to be cough (32.2\%), fever (29.5\%), sore throat (14.3\%), rhinorrhea (10.3\%) and shortness of breath (5.3\%), whereas anosmia and ageusia only constituted a small minority of cases $(2.8 \%$ and $0.7 \%$, respectively $)^{16}$. In addition, a single-centre Malaysian study of the health records of 199 COVID-19 patients reported only $6.25 \%$ of cases experiencing anosmia ${ }^{17}$. By comparison, a telephone questionnaire study from another single centre Malaysian study of 145 patients reported the prevalence of olfactory dysfunction and dysgeusia to be $21.4 \%$ and $23.4 \%$ respectively ${ }^{18}$. The discrepancy between our findings and these previous studies is likely because self-reporting is more sensitive in identifying symptom-based conditions compared to physician-reporting in health records ${ }^{19-21}$. The rarity of patients reporting parosmia and phantosmia in our study was consistent with the findings of Parma et al. ${ }^{8}$, although other studies have reported higher prevalence of parosmia and phantosmia ${ }^{5,22}$.

The findings of chemosensory self-ratings revealed that COVID-19 chemosensory loss in our cohort was not only confined to smell and taste but also involved chemesthesis. While the proportion of smell and taste loss in our cohort is higher than that observed in other Asian studies ${ }^{9-11}$, its magnitude and extent is not as marked as that reported by Parma et $\mathrm{al}^{8}$ and other studies involving predominantly Caucasian populations $\mathrm{s}^{3-7}$. Possible reasons for this may include the influence of cultural context and geographical location on the awareness and perception of smell and taste ${ }^{23-25}$. In addition, the D614G mutation of the coronavirus spike protein and ethnic differences in the frequency of variants of the virus-binding entry proteins (ACE-2 and TMPRSS2) have been proposed to facilitate virus entry in the olfactory epithelium and taste buds, thus increasing the likelihood of smell and taste disturbance ${ }^{12,13}$. A recent systematic review and meta-analysis found that South Asian populations infected predominantly with G614 virus had a much higher prevalence of anosmia compared with the same ethnic population infected mostly with the D614 strain, suggesting that D614G mutation is a major contributing factor that increases the prevalence of anosmia in COVID-1926. In a study on predominantly South Asian foreign workers with mild or no symptoms at a COVID-19 community isolation facility in Singapore, the prevalence of anosmia and ageusia was $3.0 \%$ and $2.6 \%$ respectively ${ }^{27}$. Retrospective analysis of publicly-available SARS-CoV-2 genome sampled from this population found a predominance of D614 strain, supporting the hypothesis of D614G mutation-mediated increase in the prevalence of anosmia ${ }^{28}$. While the majority of early cases of COVID-19 in Malaysia between February to April 2020 involved the SARS-CoV-2 lineage B.6 that did not harbour the D614G mutation $^{29}$, the rapid spread of new cases in Malaysia between the months of May to December 2020 was found to be due to an increase in frequency of viral strains harbouring the D614G mutation ${ }^{30}$. Hence, it is plausible that the higher proportion of chemosensory disturbances in our cohort relative to other studies from Asia may also reflect the increasing frequency of viral strains harbouring the D614G mutation during the period of our study, although further work is needed to verify this hypothesis.

The association between loss of appetite and gastrointestinal disturbances with loss of smell or taste on multivariate analysis is congruent with observations from large-scale population studies of COVID-19 symptoms that demonstrated association between anosmia with loss of appetite and gastrointestinal symptoms $s^{6,31,32}$. The physiological relevance of these findings is highly plausible since our appetite is tightly linked to smell and taste, and may reflect concomitant SARS-CoV-2 viral infection of the olfactory and gastrointestinal tract epithelia ${ }^{33,34}$.

Our findings from the principal component analysis suggest that factors other than nasal congestion underlie most of the chemosensory changes, and that sensorineural impairment was likely the dominant mechanism in our cohort with only a small proportion of smell and taste loss may be attributed to nasal congestion. Importantly, chemesthesis loss was independent of nasal congestion. Olfactory dysfunction has been proposed to be due to conductive loss from mucosal obstruction of the olfactory cleft ${ }^{35}$ or sensorineural impairment from the direct effect of the virus on olfactory epithelium ${ }^{36-40}$. In particular, SARS-CoV-2 viral infection has been shown to cause anosmia by infecting the non-neuronal sustentacular cells in the olfactory epithelium that express ACE-2, the receptor required for viral entry into the cell $^{36,37}$. Other mechanisms have implicated damage to the olfactory neurons from pro-inflammatory cytokines ${ }^{39}$ and disruption of signalling from olfactory sensory neurons to the olfactory bulb ${ }^{40}$. However, whether loss of neuronal cells actually occurs in COVID-19 and causes anosmia is currently controversial due to a lack of convincing evidence for this ${ }^{41}$.

Mechanisms for taste loss is less clear, since taste is closely linked to smell and nasal congestion. However, SARS-CoV-2 may infect taste chemoreceptor cells since ACE-2 is expressed on tongue keratinocytes ${ }^{42,43}$ or $\mathrm{cra}^{-}$ nial nerves responsible for gustation (cranial nerves VII, IX and X) although evidence for this is lacking. Loss of chemesthesis have been hypothesised to be due to viral infection of the trigeminal nerve although, again, evidence for this is lacking ${ }^{8}$. Our findings support a dominant role for sensorineural mechanisms in SARS-CoV-2-related loss of smell, taste, and chemesthesis.

The major limitation of our study is the reliance on self-reporting of chemosensory function, which is subjective. Objective assessment of olfactory dysfunction in COVID-19 have been reported using a number of psychophysical tests such as Sniffin' Sticks test ${ }^{44}$, Connecticut Chemosensory Research Centre orthonasal olfaction test ${ }^{45}$, and the University of Pennsylvania Smell Identification Test ${ }^{46}$. Likewise, gustatory dysfunction in COVID-19 have also been studied using objective tools such as a four-item taste test (sweet, sour, salty, and bitter $)^{45}$ and taste-strips impregnated with four taste qualities ${ }^{47}$. Nonetheless, self-reporting of chemosensory 
function is still widely used with reasonable accuracy rates between 70 and $80 \%^{48,49}$, and may be useful for remote assessment of patients in the setting of a pandemic. Moreover, there is evidence to suggest that objective testing is not always the most sensitive approach in detecting smell and taste loss in COVID-19. Boscutti et al. recently published a systematic review and meta-analysis of all observational studies reporting the prevalence and longitudinal trajectories of olfactory and gustatory disorders in COVID-19 using patient self-reporting and objective psychophysical tests ${ }^{50}$. They found higher prevalence from self-reporting compared to psychophysical testing in some studies whereas the opposite was true for other studies, leading them to conclude that psychophysical testing was not always the most sensitive measure ${ }^{50}$. The replicability of tests across different countries has been suggested as a possible confounding factor ${ }^{50}$. Therefore, while objective tests for smell and taste are important, there is value in studying the chemosensory disorder in COVID-19 using self-reporting. Other limitations of our study include recall bias, the use of convenient sampling, and the lack of validation of the translated version of the questionnaire within our Malaysian population.

We accounted for individual differences in baseline chemosensory abilities and the use of rating scales in two ways-first, our study used a within-subject design where the participants rated their abilities at different time points (before and during COVID-19). The same individual participates in all conditions, hence controlling for differences in participant characteristics. Second, we analysed the differences in ratings between the two timepoints ("during COVID-19" minus "before COVID-19"), instead of using absolute ratings. Hence, this approach precludes the need to normalise ratings to the baseline since we are not analysing the absolute values. Of note, this study design and method of analysis have been previously employed in large scale studies of chemosensory loss in COVID-19 using self-ratings ${ }^{8,51,52}$.

Our study also unveils opportunities to improve our understanding of COVID-19-associated chemosensory disturbances in Asian versus Caucasian populations. Future studies should compare these self-reported findings to culturally-adapted smell identification tests, such as a recently developed Malaysian version of Sniffin' Stick Smell Identification test ${ }^{53}$, psychophysical tests of smell and taste, and imaging to assess the patency of the olfactory clefts and nasal cavity. Recent loss of smell has been suggested to be the best predictor of COVID-19 diagnosis ${ }^{51}$, and therefore, it would be of significant clinical importance to determine whether or not this is the case in Asian populations.

\section{Conclusion}

In summary, our study reveals widespread loss of smell, taste, and chemesthesis in Malaysian COVID-19 patients that manifested as early symptoms of infection. These chemosensory losses largely cannot be accounted for by nasal blockage, suggesting a predominantly sensorineural aetiology. These findings challenge earlier reports that smell and taste loss in COVID-19 are less common among Asians, suggesting that these symptoms may be more common than previously thought and may be related to the infectivity of the SARS-CoV-2 strains.

\section{Data availability}

The datasets generated during and/or analysed during the current study are available from the corresponding author on reasonable request.

Received: 8 August 2021; Accepted: 21 January 2022

Published online: 08 February 2022

\section{References}

1. Guan, W. et al. Clinical characteristics of Coronavirus Disease 2019 in China. N. Engl. J. Med. 382, 1708-1720 (2020).

2. Goyal, P. et al. Clinical characteristics of Covid-19 in New York City. N. Engl. J. Med. 382, 2372-2374 (2020).

3. Giacomelli, A. et al. Self-reported olfactory and taste disorders in patients with Severe Acute Respiratory Coronavirus 2 Infection: A cross-sectional study. Clin. Infect. Dis. 71, 889-890 (2020).

4. Spinato, G. et al. Alterations in smell or taste in mildly symptomatic outpatients with SARS-CoV-2 infection. JAMA 323, 2089-2090 (2020).

5. Lechien, J. R. et al. Olfactory and gustatory dysfunctions as a clinical presentation of mild-to-moderate forms of the coronavirus disease (COVID-19): A multicenter European study. Eur. Arch. Otorhinolaryngol. 277, 2251-2261 (2020).

6. Menni, C. et al. Real-time tracking of self-reported symptoms to predict potential COVID-19. Nat. Med. 26, 1037-1040 (2020).

7. Kaye, R., Chang, C. W. D., Kazahaya, K., Brereton, J. \& Denneny, J. C. COVID-19 anosmia reporting tool: Initial findings. Otolaryngol. Neck Surg. 163, 132-134 (2020).

8. Parma, V. et al. More than smell-COVID-19 is associated with severe impairment of smell, taste, and chemesthesis. Chem. Senses 45, 609-622 (2020).

9. Mao, L. et al. Neurologic manifestations of hospitalized patients with Coronavirus Disease 2019 in Wuhan China. JAMA Neurol. 77, 683-690 (2020).

10. Lee, Y., Min, P., Lee, S. \& Kim, S. W. Prevalence and duration of acute loss of smell or taste in COVID-19 patients. J. Korean Med. Sci. 35, e174 (2020).

11. Tham, A. C. et al. Olfactory taste disorder as a presenting symptom of COVID-19: A large single-center Singapore study. Eur. Arch. Otorhinolaryngol. 278, 1853-1862 (2021).

12. Butowt, R., Bilinska, K. \& Von Bartheld, C. S. Chemosensory dysfunction in COVID-19: Integration of genetic and epidemiological data points to D614G spike protein variant as a contributing factor. ACS Chem. Neurosci. 11, 3180-3184 (2020).

13. Von Bartheld, C. S., Butowt, R. \& Hagen, M. M. Prevalence of chemosensory dysfunction in COVID-19 patients: A systematic review and meta-analysis reveals significant ethnic differences. ACS Chem. Neurosci. 11, 2944-2961 (2020).

14. Lemeshow, S., Hosmer, D. W., Klar, J. \& Lwanga, S. K. Adequacy of sample size in health studies. (John Wiley \& Sons, Inc., 1990).

15. Lê, S., Josse, J. \& Husson, F. FactoMineR: An R package for multivariate analysis. J. Stat. Softw. 25, 1-18 (2008).

16. Sim, B. L. H. et al. Clinical characteristics and risk factors for severe COVID-19 infections in Malaysia: A nationwide observational study. Lancet Reg. Heal. West. Pacific 4, 100055 (2020).

17. Ng, B. H. et al. COVID-19 detected from targeted contact tracing, attempting to see the pattern in random happenings: Early lessons in Malaysia. Med. J. Malaysia 75, 582-584 (2020). 
18. Ramasamy, K., Saniasiaya, J. \& Abdul Gani, N. Olfactory and gustatory dysfunctions as a clinical manifestation of Coronavirus Disease 2019 in a Malaysian tertiary center. Ann. Otol. Rhinol. Laryngol. 130, 513-519 (2021).

19. Violán, C. et al. Comparison of the information provided by electronic health records data and a population health survey to estimate prevalence of selected health conditions and multimorbidity. BMC Public Health 13, 251 (2013).

20. Basch, E. et al. Patient versus clinician symptom reporting using the National Cancer Institute Common Terminology Criteria for Adverse Events: Results of a questionnaire-based study. Lancet Oncol. 7, 903-909 (2006).

21. Fromme, E. K., Eilers, K. M., Mori, M., Hsieh, Y. C. \& Beer, T. M. How accurate is clinician reporting of chemotherapy adverse effects? A comparison with patient-reported symptoms from the Quality-of-Life Questionnaire C30. J. Clin. Oncol. 22, 3485-3490 (2004).

22. Hopkins, C. et al. Six month follow-up of self-reported loss of smell during the COVID-19 pandemic. Rhinology 59, 26-31 (2021).

23. Majid, A., Burenhult, N., Stensmyr, M., De Valk, J. \& Hansson, B. S. Olfactory language and abstraction across cultures. Philos. Trans. R. Soc. B Biol. Sci. 373, 20170139 (2018).

24. Majid, A. Human olfaction at the intersection of language, culture, and biology. Trends Cogn. Sci. 25, 111-123 (2021).

25. Baharuddin, A. R. \& Sharifudin. The impact of geographical location on taste sensitivity and preference. Int. Food Res. J. 22, 731-738 (2015).

26. Von Bartheld, C. S., Hagen, M. M. \& Butowt, R. The D614G virus mutation enhances anosmia in COVID-19 patients: Evidence from a systematic review and meta-analysis of studies from South Asia. ACS Chem. Neurosci. 12, 3535-3549 (2021).

27. Soh, S. H. L. et al. Prevalence of olfactory and taste dysfunction in COVID-19 patients: A community care facility study. Eur. Arch. Otorhinolaryngol. 278, 3375-3380 (2021).

28. See, A., Ko, K. K. K. \& Toh, S. T. Epidemiological analysis in support of hypothesis that D614G virus mutation is a major contributing factor to chemosensory dysfunction in COVID-19 patients. Eur. Arch. Otorhinolaryngol. 278, 3595-3596 (2021).

29. Chong, Y. M. et al. SARS-CoV-2 lineage B.6 was the major contributor to early pandemic transmission in Malaysia. PLoS Negl. Trop. Dis. 14, 1-12 (2020).

30. Mat Yassim, A. S. et al. COVID-19 outbreak in Malaysia: Decoding D614G mutation of SARS-CoV-2 virus isolated from an asymptomatic case in Pahang. Mater. Today Proc. https://doi.org/10.1016/j.matpr.2021.02.387 (2021).

31. Sudre, C. et al. Symptom clusters in COVID-19: a potential clinical prediction tool from the COVID Symptom study app. Preprint at medRxiv https://doi.org/10.1101/2020.06.12.20129056 (2020).

32. Elliott, J. et al. Symptom reporting in over 1 million people: community detection of COVID-19. Preprint at medRxiv https://doi. org/10.1101/2021.02.10.21251480 (2021).

33. Sungnak, W. et al. SARS-CoV-2 entry factors are highly expressed in nasal epithelial cells together with innate immune genes. Nat. Med. 26, 681-687 (2020).

34. Xiao, F. et al. Evidence for gastrointestinal infection of SARS-CoV-2. Gastroenterology 158, 1831-1833.e3 (2020).

35. Eliezer, M. et al. Sudden and complete olfactory loss of function as a possible symptom of COVID-19. JAMA Otolaryngol. Neck Surg. 146, 674-675 (2020).

36. Bilinska, K., Jakubowska, P., Von Bartheld, C. S. \& Butowt, R. Expression of the SARS-CoV-2 entry proteins, ACE2 and TMPRSS2, in cells of the olfactory epithelium: Identification of cell types and trends with age. ACS Chem. Neurosci. 11, 1555-1562 (2020).

37. Brann, D. H. et al. Non-neuronal expression of SARS-CoV-2 entry genes in the olfactory system suggests mechanisms underlying COVID-19-associated anosmia. Sci. Adv. 6, 5801-5832 (2020).

38. Zheng, J. et al. COVID-19 treatments and pathogenesis including anosmia in K18-hACE2 mice. Nature 589, 603-607 (2021).

39. Cazzolla, A. P. et al. Taste and smell disorders in COVID-19 patients: Role of interleukin-6. ACS Chem. Neurosci. 11, 2774-2781 (2020).

40. Rodriguez, S. et al. Innate immune signaling in the olfactory epithelium reduces odorant receptor levels: Modeling transient smell loss in COVID-19 patients. Preprint at medRxiv https://doi.org/10.1101/2020.06.14.20131128 (2020).

41. Butowt, R., Meunier, N., Bryche, B. \& von Bartheld, C. S. The olfactory nerve is not a likely route to brain infection in COVID-19: A critical review of data from humans and animal models. Acta Neuropathol. 141, 809-822 (2021).

42. Sato, T. et al. Expression of ACE2 and TMPRSS2 proteins in the upper and lower aerodigestive tracts of rats: Implications on COVID 19 Infections. Laryngoscope 131, E932-E939 (2021).

43. Xu, H. et al. High expression of ACE2 receptor of $2019-\mathrm{nCoV}$ on the epithelial cells of oral mucosa. Int. J. Oral Sci. 12, 8 (2020).

44. Lechien, J. R. et al. Loss of smell and taste in 2013 european patients with mild to moderate covid-19. Ann. Intern. Med. 173, $672-675(2020)$.

45. Vaira, L. A. et al. Olfactory and gustatory function impairment in COVID-19 patients: Italian objective multicenter-study. Head Neck 42, 1560-1569 (2020).

46. Moein, S. T. et al. Smell dysfunction: A biomarker for COVID-19. Int. Forum Allergy Rhinol. 10, 944-950 (2020).

47. Hintschich, C. A. et al. Psychophysical tests reveal impaired olfaction but preserved gustation in COVID-19 patients. Int. Forum Allergy Rhinol. 10, 1105-1107 (2020).

48. Lötsch, J. \& Hummel, T. Clinical usefulness of self-rated olfactory performance-a data science-based assessment of 6000 patients. Chem. Senses 44, 357-364 (2019).

49. Hoffman, H. J., Rawal, S., Li, C. M. \& Duffy, V. B. New chemosensory component in the US National Health and Nutrition Examination Survey (NHANES): first-year results for measured olfactory dysfunction. Rev. Endocr. Metab. Disord. 17, 221-240 (2016).

50. Boscutti, A. et al. Olfactory and gustatory dysfunctions in SARS-CoV-2 infection: a systematic review. Brain, Behav. Immun. - Heal. 15,100268 (2021).

51. Gerkin, R. C. et al. Recent smell loss is the best predictor of COVID-19 among individuals with recent respiratory symptoms. Chem. Senses 46, bjaa081 (2021).

52. Cecchetto, C. et al. Assessing the extent and timing of chemosensory impairments during COVID-19 pandemic. Sci. Rep. 11, $17504(2021)$.

53. Sai-Guan, L., Husain, S., Zahedi, F. D., Ahmad, N. \& Gendeh, B. S. Cultural adaptation of Sniffin' Sticks smell identification test: the Malaysian version. Iran. J. Otorhinolaryngol. 32, 213-222 (2020).

\section{Acknowledgements}

We would like to thank our patients for participating, and acknowledge all healthcare workers involved in the management of COVID-19 cases in Malaysia. We thank the Director-General of Health, Malaysia for granting permission to publish this article. We are grateful to John R. Griffiths, DPhil, FRCP (Cancer Research UK Cambridge Institute, University of Cambridge, United Kingdom) for his comments on the paper.

\section{Author contributions}

S.-H.L., Z.X.Y., I.S.S., N.G., S.A.S., and S.S.M.H. conceived and designed the study. All authors were involved in the acquisition, analysis, and interpretation of data. S.-H.L., S.A.S., and C.Y.F. performed the statistical analyses. S.A.S., L.L.L., S.B.S.A., T.M.I.T.K., J.S., M.Z., C.Y.W., H.H.C., S.Y., D.M., P.R.D., H.B.K., Z.S., M.M., H.S., H.G.L., S.D., N.A.M., A.M., M.N.I., C.P., T.S.C., E.K.K., C.L.L., L.P.F.L., N.Z.Z., M.Z.I., and S.A.W. provided 
administrative, technical, or material support. S.-H.L. drafted the manuscript with critical input from C.Y.F. and S.A.S. All authors reviewed the manuscript and approved the final version. S.-H.L. and S.S.M.H. jointly supervised the study.

\title{
Competing interests
}

The authors declare no competing interests.

\section{Additional information}

Supplementary Information The online version contains supplementary material available at https://doi.org/ 10.1038/s41598-022-06029-6.

Correspondence and requests for materials should be addressed to S.-H.L.

Reprints and permissions information is available at www.nature.com/reprints.

Publisher's note Springer Nature remains neutral with regard to jurisdictional claims in published maps and institutional affiliations.

\begin{abstract}
(c) (i) Open Access This article is licensed under a Creative Commons Attribution 4.0 International License, which permits use, sharing, adaptation, distribution and reproduction in any medium or format, as long as you give appropriate credit to the original author(s) and the source, provide a link to the Creative Commons licence, and indicate if changes were made. The images or other third party material in this article are included in the article's Creative Commons licence, unless indicated otherwise in a credit line to the material. If material is not included in the article's Creative Commons licence and your intended use is not permitted by statutory regulation or exceeds the permitted use, you will need to obtain permission directly from the copyright holder. To view a copy of this licence, visit http://creativecommons.org/licenses/by/4.0/.
\end{abstract}

(c) The Author(s) 2022

\section{Malaysian COVID-19 Anosmia Research Group}

Nurul Asyikin Bachok ${ }^{30}$, Linger Sim ${ }^{8}$, Tiana $\mathrm{Ti}^{9}$, Teng Huei Lee ${ }^{8}$, Siti Nurul Aliaa Mohd Nor ${ }^{8}$, Kim Siang Tay ${ }^{9}$, Abirami Gouri Pagan ${ }^{9}$, Anura Aman ${ }^{10}$, Kamariah Mohamed Awang ${ }^{10}$, Jamal Nasser Salleh ${ }^{10}$, Harridas Manickam ${ }^{10}$, Nursyamimi Mohamad Zaki ${ }^{10}$, Cheng Keat Moh ${ }^{12}$, Ruthran Thurairajuj ${ }^{12}$, Ho Hwee Yee ${ }^{12}$, Nabilah Abd Aziz ${ }^{12}$, Rosdi Ramli ${ }^{14}$, Rosli Mohd Noor ${ }^{14}$, Anilawati Mat Jelani ${ }^{15}$, Mohd Fakri Alimi Mustapha ${ }^{14}$, Abdul Aziez Ab Aziry ${ }^{14}$, Kribananthan Lohanadan ${ }^{16}$, Siti Farhana Abdul Razak ${ }^{16}$, Yen Tsen Saw ${ }^{17}$, Jason Henn Leong Kong ${ }^{17}$, Carren Sui Lin Teh ${ }^{18}$, Kuldip Kaur Prem Singh ${ }^{19}$, Arvindan Karunakaran ${ }^{18}$, Nesha Rajendram ${ }^{18}$, Nik Khairani Nik Mohd ${ }^{20}$, Nurul Amilin Ja'afar ${ }^{20}$, Siti Sarah Che Mohd Razali ${ }^{20}$, Shamesh Baskaran $^{22}$, Farrah Hani Hassan ${ }^{24}$, Kalaiselvi Thuraisingam ${ }^{24}$, Hanisah Hithayathullah ${ }^{24}$, Prempreet Kaur Manjeet Singh ${ }^{24}$, Shen-Han Lee ${ }^{1}$, Nadiah Hanim Zainul ${ }^{4}$, Man Chek Ooi ${ }^{1}$, Siti Aishah Mahadziri ${ }^{1}$, Nurul Afifah Mohd Yusoff ${ }^{1}$, Anees Fatimah Mohamad Illiayas ${ }^{1}$, Yi Shan $\mathrm{Tan}^{4}$, Ibtisam Ismail2, Huan Keat Chan², Jeyasakthy Saniasiaya ${ }^{31}$, Tiang Koi $\mathrm{Ng}^{32}$, Kuganathan Ramasamy ${ }^{31}$ \& Fatin Farha Mohd Saifuddin ${ }^{29}$

${ }^{30}$ Hospital Enche' Besar Hajjah Khalsom, Johor, Malaysia. ${ }^{31}$ Department of Otorhinolaryngology, Hospital Tuanku Ja'afar, Negeri Sembilan, Malaysia. ${ }^{32}$ Department of Medicine, Hospital Tuanku Ja'afar, Negeri Sembilan, Malaysia. 